\title{
Evliyâ Çelebi en Grèce, de la Thrace à l'Attique
}

Evliyâ Çelebi in Greece, from Thrace to Attika

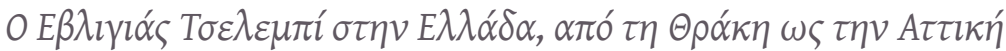

Joëlle Dalègre

\section{(2) OpenEdition}

Journals

Édition électronique

URL : https://journals.openedition.org/ceb/3990

DOI : $10.4000 /$ ceb.3990

ISSN : 2261-4184

Éditeur

INALCO

Édition imprimée

Pagination : 89-97

ISBN : 978-2-85831-205-4

ISSN : 0290-7402

Référence électronique

Joëlle Dalègre, "Evliyâ Çelebi en Grèce, de la Thrace à I'Attique », Cahiers balkaniques [En ligne], 41 |

2013, mis en ligne le 19 mai 2013, consulté le 06 juillet 2021. URL : http://journals.openedition.org/ ceb/3990 ; DOI : https://doi.org/10.4000/ceb.3990

Ce document a été généré automatiquement le 6 juillet 2021.

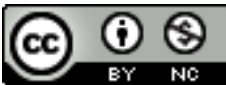

Cahiers balkaniques est mis à disposition selon les termes de la Licence Creative Commons Attribution - Pas d'Utilisation Commerciale 4.0 International. 


\title{
Evliyâ Çelebi en Grèce, de la Thrace à l'Attique
}

\author{
Evliyâ Çelebi in Greece, from Thrace to Attika

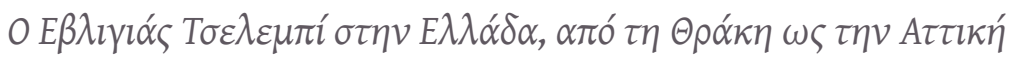

Joëlle Dalègre

\section{La source}

1 Cet article n'a utilisé qu'un tome d'un ouvrage en grec qui présente des extraits des voyages d'Evliyâ Çelebi, en allant de la Thrace jusqu'en Attique et en les classant du Nord au Sud sans suivre la chronologie.

\section{L'ouvrage}

2 Nikos Cheiladakis "Evliyâ Çelebi, Voyage en Grèce " tome 1 (le 2 concerne les îles)

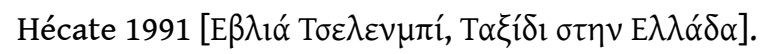

3 L'auteur dit avoir réuni des traductions grecques devenues introuvables ou incompréhensibles, car en langue trop puriste.

4 1. «Les chapitres concernant Athènes dans Evliyâ Çelebi » Hellenika, 3.1930, trad. D

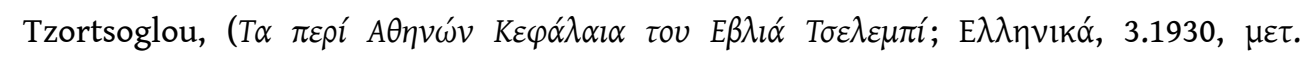
$\Delta . T \zeta \omega \rho \tau \zeta o \gamma \lambda \circ u ́)$.

5 2. «Evliyâ Çelebi en terres grecques " Hellenika 3.1931, qui complète et corrige le

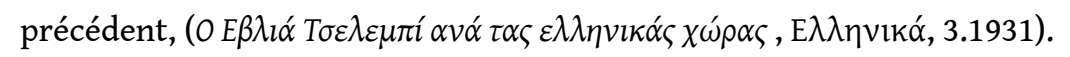

6 3. " La Thrace occidentale selon Evliyâ Çelebi » Thrakika 4-1033 et 5-1934, trad.

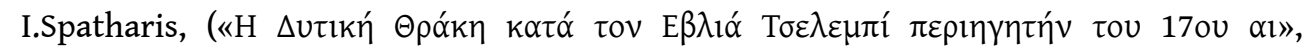

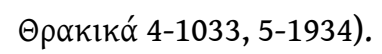

7 4. « Pages de la vie de la vieille Turquie des Janissaires (selon la description du voyageur turc du XvII ${ }^{\mathrm{e}}$ siècle, Evliyâ Çelebi » trad. A. A. Pallis, rééd chez Hécate en 1990. ( $\Sigma \varepsilon \lambda i \delta \varepsilon \varepsilon$ 


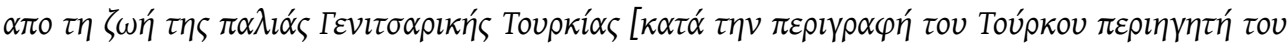

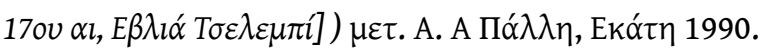

8 5. «La Grèce selon Evliyâ Çelebi » Journal de la société d'études byzantines, 14-1938,

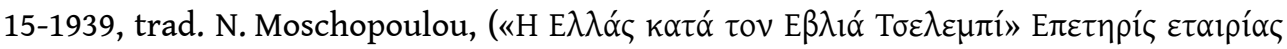

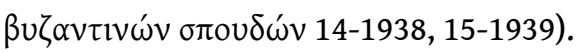

6. "Voyage d'un Turc en Épire", Ipirotika Grammata, 1944, trad. A. Kechayoglou et

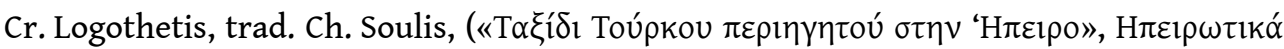
үра́ $\mu \mu \alpha \tau \alpha, 1944)$

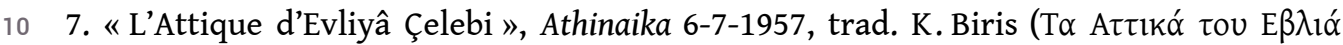

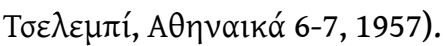

11 8. "L'Attique d'Evliyâ Çelebi, Athènes et ses environs au XVII' siècle », Trad. K. Biris, Athènes,

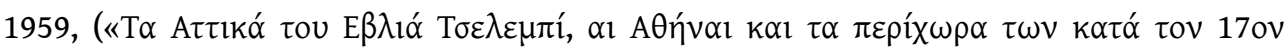

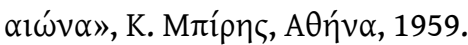

12 9. «L'Eubée d'Evliyâ Çelebi » Evoiki Bibliographia, 1959, trad. I. Argyropoulou et

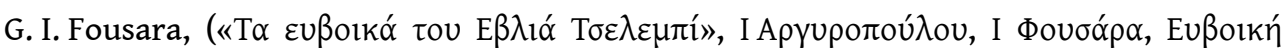

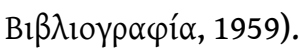

13 10. «Le voyage d'Evliyâ Çelebi en Grèce continentale ", Journal de la société des études de

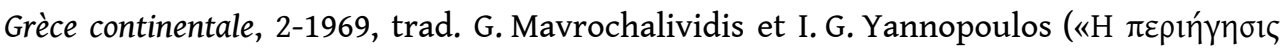

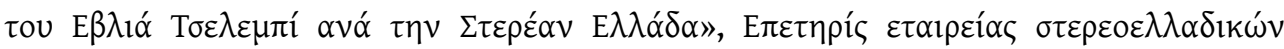

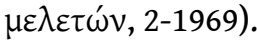

\section{Le traducteur}

14 Nikos Cheiladakis, le responsable de cette publication vit à Salonique, il a étudié l'économie en Grèce et le turc à Paris. Il écrit dans des quotidiens régionaux et nationaux en Grèce, collabore avec des radios privées, intervenant toujours à propos des relations gréco-turques; il a publié 18 livres, toujours en relation avec la Turquie contemporaine, dans l'ensemble tous assez hostiles aux Turcs. Dans l'introduction de ce recueil, il insiste sur l'importance des textes de Çelebi et déplore qu'ils ne soient pas assez connus en Grèce ; il affirme faire œuvre "nationale » en réunissant les extraits qu'il présente et en retraduisant des articles anciens qui sont dans une langue aujourd'hui incompréhensible ou introuvables. Il a publié également un $2^{\mathrm{e}}$ tome, consacré aux voyages dans les îles et en Crète que, malheureusement, je n'ai pu obtenir à temps. Faute de pouvoir lire Çelebi dans le texte, je suis tributaire des choix de cet auteur, des coupes qu'il a faites parfois et de son classement. Il s'agit donc de la Grèce de Çelebi, telle qu'elle est présentée par un Grec au lecteur grec.

\section{Les textes choisis}

Çelebi a, en 1630, voyagé en Thrace au départ de la capitale, participé à l'expédition de Crète en 1645 et de nouveau en 1667 (le $8^{\mathrm{e}}$ livre) en ayant, dans les deux cas, traversé une partie de la Grèce continentale sur le parcours; il a abordé le Dodécanèse au retour de la Mecque.

16 Les textes ont été classés par Cheiladakis en huit chapitres, Thrace occidentale, Macédoine orientale, Salonique, Grèce continentale orientale, Eubée, Athènes et 
Attique, Grèce continentale, Épire. Dans l'itinéraire dit "Grèce centrale ouest », Çelebi a sans doute inséré des renseignements qu'il a reçus sans y être réellement allé, ainsi pour Karpenissi (localisation erronée) et Amphissa (port de mer ?).

17 Une autre communication étant consacrée à Athènes, j'ai choisi de développer les grandes lignes observées dans ce livre, puis de regarder plus en détail sa présentation de Salonique, en laissant de côté le chapitre athénien.

\section{Un guide pour esprits curieux}

18 À première lecture, le texte se présente comme un guide adapté aux intérêts du $\mathrm{XVII}^{\mathrm{e}}$ siècle. On trouve donc de façon systématique l'étymologie du lieu, son histoire, sa situation administrative, fiscale et militaire détaillée et la description de sa forteresse. On passe ensuite aux quartiers extra-muros, nombre de mahalles et de maisons, répartition par religions, le nombre des mosquées, mescids, tekkes, parfois églises, medreses, mektebs, imarets, hamams et hans et les lieux saints éventuels. Puis vient le tour des commerces, nombre, spécialité, bedesten couvert ou non, dallé ou non; on trouve enfin les conditions de vie: qualité des eaux des fleuves et des fontaines, spécialités agricoles ou artisanales; il relie toujours récoltes, climat et état de santé. Comme il est prêt à recueillir les anecdotes et à voir les " curiosités ", ce tableau un peu standard est émaillé de pirates, de miracles et de petites mises au point sur des sujets divers.

Les présentations historiques et étymologiques mettent en scène souvent les mêmes personnages dans un syncrétisme philosophico-religieux exemplaire.

- Les philosophes antiques qu'il cite souvent, le sage "Pythagore», Hippocrate, Socrate, Aristote, Philippe le philosophe, et les plus souvent nommés, le « divin » Platon et Ptolémée le philosophe, successeur d'Aristote au Lycée qu'il appelle Balatimos et Baltimos, celui qu'il appelle le sage Philikos, roi des Grecs, père d'Iskender (Alexandre) «le plus grand roi du monde ".

- Il attribue à Filikos la fondation de Salonique, de Kavala, de la forteresse de Naupacte, et à Alexandre, la fondation de la plupart des forteresses de Grèce du Nord et même le percement du passage de l'Euripe entre l'Eubée et continent.

- Le $3^{\text {e }}$ personnage souvent cité est Salomon, lui aussi «le sage», il a fait enlever par ses néréides la fille si belle du roi de Saba, Belkis, ils sont ensuite tous deux transportés par les vents à Sfakia, en Crète, puis en Attique (Kallithéa) et enfin, Belkis depuis le ciel, choisit l'emplacement de Salonique où il lui construit un palais à l'emplacement de Yedi Kule. Interviennent également Roboam le fils de Salomon qui, selon certains dit-il, serait le père du roi Filikos. Le prophète Zacharias, arrière-petit-fils de Salomon, ayant été martyrisé à mort par des juifs, le philosophe Baltimos aurait conduit une expédition punitive dans la région d'Alexandrette, tuant 100000 juifs, il serait enterré dans une église du mont Athos construite, aussi, par Alexandre ! Et à Salonique, pour la même raison, les juifs auraient été chassés de Palestine par un prince kurde cette fois et venus se réfugier.

Ses étymologies relèvent également de la mythologie, un Kavals fils de Filikos pour Kavala, Demos et Doukas pour Didimoteicho (dont il nous décrit pourtant le double mur " dydimoteicho »), une reine Ayasof qui aurait construit les bâtiments du même nom à Salonique, à Istanbul et à Trébizonde, Lamia, Izdin à cause de Zidina la fille d'un roi, Livazia fille du divin Platon à l'origine de Livadia, l'Eubée "Evripos" à cause d'un prince du même nom... 
21 Son historique est beaucoup plus exact quand il s'agit de la conquête turque puisque, pour chaque lieu, il indique le nom du conquérant et les conditions du succès, précisant que dans certains cas, par la soumission sans résistance, les habitants ont obtenu une exemption d'impôts ou le droit d'habiter la forteresse.

Les descriptions des forteresses sont très détaillées; dans ce domaine comme dans les autres, Çelebi compare toujours avec ce qu'il connaît ailleurs, aussi grande que celle de..., plus elle, plus petite, etc. Il nous donne le périmètre, l'état des murailles, le nombre des portes, l'épaisseur et la hauteur des murs, la présence ou non d'un fossé, l'état des canons, l'état intérieur de la forteresse, tout cela pour dire si cette forteresse est facile à défendre ou non. Les plus grandes dans le secteur considéré sont, en dehors de Salonique, Kavala (plus de 3000 pieds de tour), Iannina et Chalcis et, grande source d'admiration, celle de Naupacte, quasiment imprenable. Mais là, il ne cite pas la bataille de Lépante, pourtant encore assez récente.

La description des quartiers d'habitation comprend le nombre des mahalles intra et extra-muros. Il parle de « fidèles et infidèles ", jamais de " musulmans », mais pour les « infidèles », détaille les Rums, les Latins, parfois les Francs et les Arméniens, puis les Juifs et les Tsiganes; quand le nombre est plus important, il précise Rums, Bulgares et Serbes, ce qui signifie qu'il n'assimile pas tous les orthodoxes aux Rums, qu'une différenciation existe déjà (le cas se présente pour plusieurs villages «bulgares » en Thrace et Macédoine). Pour présenter une construction byzantine, il dit « du temps des Infidèles ", et pour une construction antique, il parle "de Rums idolâtres" ou de Yunan, le "Yunan Iskender». Il présente la population des grands centres comme parlant très bien le roméique et le turc, à Salonique s'y ajoutent le bulgare et la langue hébraïque, dit-il ; dans certains villages de Macédoine, il entend du grec et du bulgare. À l'occasion de son passage à Komotini, il consacre un long développement aux Tziganes-Coptes, dit-il et à leur langue. À Domokos (op.cit. p 130) :

Je n'ai pas pu comprendre quel genre de musulmans ils sont. Ils mangent et boivent toute la journée avec les infidèles Rums et font la fête avec eux, ils ne sont musulmans que de nom en réalité, c'est une race de rayas qui est exemptée de l'obligation de payer le haratch.

24 Les plus grandes villes sur ce trajet sont - en dehors de Salonique - sont Serrès et Ioannina, l'une a 10 mahallas chrétiens et juifs sur un total de 40 et 4000 maisons; Ioannina compte également 4000 maisons de très belle qualité, Komotini a 4000 maisons. Ce sont des villes bien équipées: Serrès possède 91 lieux de culte musulman, 710 fontaines, 2060 puits dans les maisons, 1060 hammams privés, 17 hans, 7 asiles pour célibataires, églises et il conclut :

une pareille ville en Roumélie, il est impossible d'en rencontrer. Seulement peutêtre en Perse ou aux pays des Infidèles, où il y a des villes comme Dunkerque, Danemark, Londres, Amsterdam qui ont d'aussi belles allées d'arbres! Heureuse la ville et ses habitants dans les siècles des siècles !

Serrès est, dit-il, une ville connue de tous les voyageurs et cartographes, un nœud de communications, elle a 10 mahallas chrétiens, Rums, Arméniens, Latins, Bulgares, Serbes et aussi Juifs, riches commerçants avec de très belles maisons sur un total de 40 , les 30 autres aux musulmans, 4000 maisons d'un ou deux étages, avec chacune puits ou citerne. À Ioannina, il dénombre aussi 4000 maisons, 4 mahallas intra muros et 37 en dehors, au total 14 chrétiens, 4 juifs et un Tsigane. Les maisons ont des difficultés pour l'approvisionnement en eau, mais sont entourées d'un grand jardin, de vignes et d'un potager, le rez-de-chaussée en pierre et la partie supérieure en bois. Ioannina a un 
sérail de plus de 400 pièces. Les grandes villes sont au centre de régions agricoles dont il vante les richesses comme Komotini et, à Serrès, les céréales, fruits secs, des buffles si costauds, plus que ceux du Kurdistan, de Van, d'Adana ou de Tarse, et de grands centres commerciaux comme Ioannina où l'on rencontre des Arabes, des Perses, des Francs.

Un homme passionné d'architecture : à propos des maisons, il précise toujours si les toits sont en tuiles ou en lauze, le nombre d'étages, il signale et admire les ouvrages d'art de qualité, aqueduc (Kavala, Livadia), ponts (Arta, pont sur le Nestos, ponts de pierre près de Serrès) et s'enthousiasme sur le détail de certaines mosquées. Ainsi à Didymoteicho, la mosquée Beyazit « la construction est couverte de plomb et le métal utilisé pour le toit a été travaillé avec une telle qualité et une telle maîtrise qu'à sa surface se reflètent toutes les couleurs de l'arc en ciel et donnant à toute la construction la couleur magique d'un caméléon. ", la mosquée Gazi Ibrahim pacha à Kavala qui dispose d'une riche décoration, comprend imaret, medressé, mektep, tekke de derviches, tous couverts en plomb « ce qui fait ressembler de loin la ville à une ville de notables. »; il est sensible aux charmes de l'urbanisation, les rues dallées de Serrès, le parc ombragé de Drama, les promenades de Ioannina, les équipements collectifs nombreux: pas de mahallas sans mosquée ou mescid, nombreux hammams publics, imaret et mektep (beaucoup moins de renseignements quand il s'agit des infidèles), pas de bourgade sans un ou plusieurs tekke.

Il est toujours fort satisfait du climat sauf en quelques villes comme Lamia, trop humide et exposée aux fièvres, et d'autres où il trouve l'été trop chaud comme Kavala, Drama ou Serrès, mais il nous décrit des lieux idylliques en altitude où les habitants de ces villes passent un été agréable. Ce climat est bénéfique :

- Didymoteicho

Le climat est tempéré. C'est pourquoi les jeunes gens et les jeunes filles de la ville (qui sont à l'âge de l'amour) ont un corps tendre et des proportions harmonieuses, leur peau est bien blanche comme de l'argent pur et sur tous les villages triomphe le rose de la santé. Le bon état physique des amants est dû à l'eau du Kizil Deli qui est de bonne qualité, légère et digestive (p. 31).

- Komotini

À cause du très bon climat, les hommes et les femmes sont ravissants et célèbres pour leur beauté. (p. 50)

- Serrès

Les habitants de Serrès ont le visage rose et plein de santé, de grâce, dans l'air merveilleux et pur et le climat tempéré. Cela les rend costauds et sains. J'ai vu des notables âgés vivre très très longtemps, parfois même quand on les croit perdus et sur le point d'expirer, justement grâce au climat de la ville, ils sont aussi forts et mobiles. (p. 84)

- Salonique

Les joues des habitants ont le rose de la santé, même leurs fronts sont roses. Les vieillards sont centenaires et atteignent 170 ans. Et à cet âge, ils peuvent ceindre l'épée, monter à cheval ou faire l'amour.

- Eubée

Leurs joues rouges et leur corps bien droit, les hommes les doivent au climat exceptionnel de la région... les jeunes gens gardent les jambes nues ce qui les rend beaux et désirables quand on voit leurs blanches chevilles. Les femmes, elles, ne sortent jamais de la maison. (p. 160)

- Athènes

Les Rums d'Athènes ont les plus belles femmes du monde. Toutes ont la peau blanche et douce, des sourcils bien dessinés, une petite bouche et des dents blanches comme des perles fines. ... Les jeunes gens sont plus beaux que les sylves 
des bois. Ils ont le visage tendre, angélique et des yeux de biche, leur grand front lumineux a la finesse des épées de Damas. Quand ils parlent doucement, ils sont encore plus beaux et attirants. Et quand tu observes le fin duvet qui blondit sur leur corps tendre, naissent immédiatement en toi les désirs les plus fous et s'allumer un désir intense.

\section{Salonique, la « petite Istanbul »}

\section{Istanbul »}

- une ville juive: des juifs massacrés et réfugiés qui s'emparent de la ville par traîtrise et dominent les chrétiens, puis acceptent sous la pression des Génois une synkyriarchia (duumvirat) de la ville, jusqu'à ce qu'Hélène, la mère du roi Constantin, les ait obligés à se soumettre. Comment ne connaît-il pas un événement qui n'a que 150 ans? Ils habitent, ditil, 56 mahallas de maisons misérables et très densément peuplées près du marché, 56 sur un total de 120 (48 aux musulmans et 16 aux infidèles chrétiens). Il précise aussi que les rues de ces quartiers sont étroites, tortueuses et sales, car elles n'ont aucun ramassage d'ordures. Il nous précise également qu'ils exercent en particulier tous les métiers du textile.

- une forteresse énorme : 3 milles de large, 2 en profondeur, en comptant les distances entre chaque porte, il parvient à un périmètre total de 11500 pas, sans compter la muraille maritime, mur de 6 coudées de large et de 25 de haut. En gros elle ne peut se comparer qu'avec Méthoni et Koroni, a des canons dont on ne peut trouver l'équivalent qu'à Rhodes ou à l'entrée des Dardanelles.

La ville a une activité commerciale très importante, grâce à son port et ses artisans. Le port très vaste, est trop exposé au sud, mais on peut s'y ancrer en profondeur ce qui compense, on peut y voir des navires « de la mer Noire, de la mer Blanche, du golfe Persique, égyptiens, algériens, de Suez, de Tripoli, de France, du Portugal, du Danemark, d'Angleterre, de Dunkerque, de Hollande, de Gênes ». Le marché égyptien, proche du débarcadère, compte 500 commerces, outre les tanneries et menuisiers voisins ; la ville compte 16 hans avec commerces, 5 grands marchés et un bedesten exceptionnel.

Le voyageur qui y entre pour la première fois a la tête qui tourne devant l'odeur, l'ambre et tous les épices qui y embaument. Dans le bedesten, au total 4400 magasins, de très riches commerçants comptent l'or et les piastres et le bruit de ces comptes provoque l'horreur des gens bons et pauvres. La foule qui se presse pour acheter ou vendre est innombrable et s'écoule dans les 300 rues, car il y en a autant que les métiers.

- Les activités de transformation y sont importantes, en particulier le tissage de tapis en coton, le tissage du drap des uniformes de Janissaires, d'un autre drap «type anglais », de serviettes éponges pour hammams, des petits tapis de laine dits « de Salonique ». La plaine autour est riche : 167 moulins à eau qui fonctionnent avec une technique unique dans l'Empire et si parfaite qu'elle fait l'admiration des Francs, les jardins produisent au total 70 types de récoltes différentes recensées aux impôts, neuf variétés de légumes, huit céréales et des légumes secs, du trèfle pour les chevaux, les collines fournissent un vin excellent (qu'il n'a pas bu !) des fruits, en particulier des cerises et l'élevage est facile. C'est donc une ville qui attire travailleurs et étrangers, d'où 70 » hôtels pour célibataires » destinés à héberger les travailleurs migrants.

Cahiers balkaniques, $41 \mid 2013$ 
Et, nantis comme étrangers de passage savent s'y distraire: 448 cafés, dit-il, 20 brasseries, 17 cafés riches qui font de la musique et des spectacles (il en signale aussi à Ioannina) qui, tous, se font beaucoup d'argent.

Le plus étrange, je ne l'ai vu en aucun autre coin du monde- est que les taverniers servent les clients et les habitués avec l'aide de femmes et de leurs enfants. Il existe une loi intangible, quel que soit son degré d'ivresse, personne ne porte la main sur une femme ou les enfants, ni ne les ennuie par des paroles vulgaires d'ivrogne. Dans ce grand désordre d'apparence, règne beaucoup d'ordre.

À ville riche, des lieux de culte hors du commun : 150 mosquées ou mescid, dont les plus belles à son goût sont en fait des constructions byzantines ou antiques, Aya Sofia, Acheiropoiitos, Aghios Dimitrios et la Rotonde. D'Aghia Sofya il dit qu'elle est égale en dimensions à celle de Trébizonde, que la chaire - un chef d'œuvre de l'art, très rare est connue de tous voyageurs par mer et par terre, comme l'une des curiosités du monde $» . .$.

« dans l'entrée de la porte extérieure il y a 9 colonnes de marbre dont chacune a une valeur égale au haradj que payent les infidèles de Roumélie !

Elle a aussi un toit en plomb qui a une rare couleur jaune dorée qui fait envie aux Juifs...

D’Eski Djami (Acheiropoiitos) il cite le minaret le plus élégant de la ville, les montants de la porte décorés de reliefs en coquillages et motifs hélicoïdaux si beaux «qu'on croirait qu'ils en sont pas œuvre humaine ", le dôme de la Rotonde, (mosquée de Hortadj sultan) est si beau qu'il fait peur, coupe la voix et éblouit, la mosquée Kasım Pacha (Aghios Dimitrios) a des marbres si beaux qu'elle attire l'attention même des nonspécialistes et qu'on ne peut trouver les mots pour la décrire, même le tekke mevlevi est encore plus réussi que celui de Beşiktaş.

Enfin il n'oublie pas que la ville est également un foyer intellectuel (le seul cas où il le dise) «de la population un tiers travaille, un tiers étudie, les autres s'occupent de religion». On compte 12 monastères, 20 écoles rabbiniques, des poètes remarquables, des lettrés en toutes disciplines, des chirurgiens habiles, de grands médecins en rapport avec ceux de l'antiquité, Hippocrate, Socrate, Galien.

\section{Conclusion}

7 Les jugements, "grand ", «bon ", « beau » sont toujours œuvre personnelle, mais au total, on comprend pourquoi l'auteur de ce recueil veut le diffuser en Grèce. La vision est largement positive, on a des maisons toujours "assez belles", des jeunes gens "roses à la peau blanche », des gens "accueillants qui font de la bonne cuisine », un climat "agréable " (sauf quelques exceptions), des récoltes variées! Le seul quartier pauvre et sale est juif et quand on rencontre des pirates chrétiens, ils ont été appelés à Moudenitsa par des habitants mécontents d'un cadi injuste et autoritaire! Le meilleur des mondes! 


\section{RÉSUMÉS}

À partir d'une traduction grecque du Livre de voyage d'Evliyâ Çelebi, cet article suit son voyage depuis la Thrace jusqu'en Attique, s'attardant sur les caractères généraux récurrents de ses descriptions et détaillant la vision de Salonique à laquelle est consacrée une large part de l'ouvrage.

From a greek translation of the Book of Evliyâ Çelebi's travels, this paper follows his journey from Thrace to Attika, focusing on the main recurrent characteristics of his descriptions and giving details of his vision of Salonica, to which is dedicated a large part of this book.

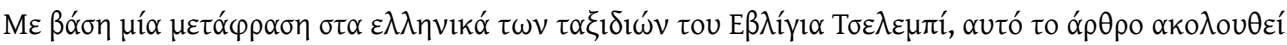

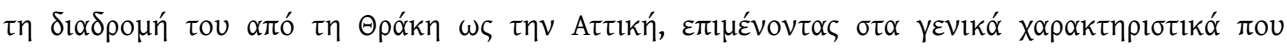

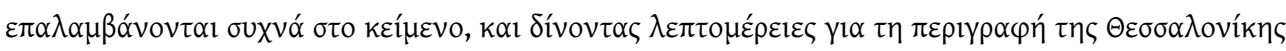

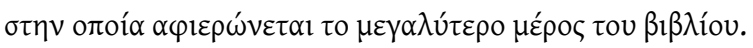

\section{INDEX}

Mots-clés : Evliyâ Çelebi (1611-1682), Evliyâ Çelebi (1611-1682), Seyahatnâme, Seyahatnâme, récit de voyage, Bedesten, forteresses

Keywords : Evliyâ Çelebi (1611-1682), Seyahatnâme, Fortresses, Architecture, Urbanism, Ottoman Empire, Salonika, Serres, seventeenth century, History of mentalities, travelogues Index géographique : Empire ottoman, Salonique, Serrès, Didymoteicho, Grèce

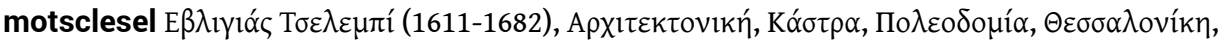

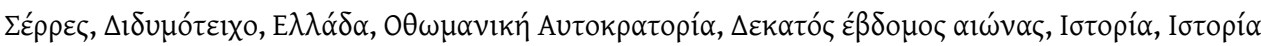

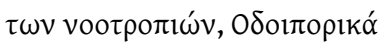
motsclestr Evliyâ Çelebi (1611-1682), Seyahatnâme, Kaleler, Mimarlık, Kent planlaması, Osmanlı İmparatorluğu, Selanik, Serres, Dimetoka, Yunanistan, Onyedinci yüzyılda, Tarih, Zihniyetlerin Tarihi, Seyahatnameler

Thèmes : Histoire, Histoire des mentalités, Architecture, Urbanisme motsclesmk ЕВЛИЈА ЧЕЛЕБИЈА, (1611-1682) СЕЈАХАТНАМЕ, ЉРХИТЕКТУРА, УРБАНО ПЛАНИРАњЕ, СОЛУН, СЕРЕЗ, ДИМЕТОКА, ТВРДИНИ, ГРЦИЈА, ОТОМАНСКАТА ИМПЕРИЈА, СЕДУЧИАЕСЕТИОТ ВЕК, ИСТОРИЈА, ИСТОРИА НА МЕНТАЛИТЕТ, ПАТЕПИСИ

Index chronologique : dix-septième siècle

\section{AUTEUR}

\section{JOËLLE DALÈGRE}

MCF-HRD, Civilisation de la Grèce moderne Inalco 